\title{
Intervención en el patrón de conducta tipo A: Un modelo interdisciplinar
}

Marta Gil-Lacruz \& Alicia Izquierdo

Universidad de Zaragoza

El patrón de conducta tipo A constituye un elemento importante en el estudio de los entornos laborales. Entre los nuevos retos de la vida moderna encontramos un mercado laboral complejo y cambiante, donde la productividad, competitividad y actuación permanente lleva implícita una importante carga de estrés, estilos de vida nocivos y riesgos evidentes para la salud en determinados grupos profesionales. El presente artículo analiza este fenómeno desde una perspectiva psicosociofisiológica y plantea las principales líneas de evaluación así como de intervención dentro de un abordaje interdisciplinar.

estilos de vida, ansiedad, enfermedad, riesgo cardiovascular, estrés laboral.

\section{Intervention in the A conduct pattern: An interdiscipliner model}

The type A imples clearly the interaction importance of the psyco, socio and physical variables involves in the behavioral explication. Nowadays the productivity, competitive an constant formation requirements in the laboral environments imply to the employers an strong amount of stress, unhealthy life styles and specif laboral risks. This article analyzes the cientific psychologycial literature about this situation researching the common point of view and their operative consequences in the laboral prevention and health promotion.

Life style, anxiety, cardiovascular illness, stress, laboral risk

Correo electrónico: mglacruz@unizar.es 


\section{ESTILOS DE VIDA: UN RETO INTERDISCIPLINAR}

Los estilos de vida constituyen una preocupación de primer orden para los sistemas sanitarios contemporáneos. Este tema presenta una mayor sensibilización pese a que nuestro nivel de salud es considerablemente superior al que ha podido caracterizar otras épocas (Montiel, 1993), debido a un buen número de factores:

- Cambios en la propia definición de salud, de la ausencia de enfermedad al bienestar biopsicosocial, la OMS reconoce en esta evolución la importancia de los parámetros culturales y sociales en la salud comunitaria (González Moro, 1990).

- Cambios en las tendencias epidemiológicas.- En la sociedad actual, una de las primeras causas de muerte son las enfermedades cardiovasculares, el cáncer y los accidentes de tráfico (Costa y López, 1986; Mira, 1990); es decir, trastornos fisiológicos derivados directa o indirectamente de los estilos de vida.

- Cambios en las representaciones sociales sobre la salud, sobre todo en los países económicamente desarrollados. Correspondería a los fenómenos que Varela y Álvarez-Uría (1989: 71) denominan: "cultura somática" y el "fetichismo del cuerpo sano", que tienden a priorizar las normas que prevalecen en otros aspectos sociales como el sobredimensionamiento de la imagen, el hedonismo y el logro.
Como plantean Maestro y Huertas (1992) es muy amplia la lista de las patologías que pueden ser incluidas en esta dinámica: las enfermedades psicosomáticas (falta de control del individuo), las enfermedades laborales (negligencia), las enfermedades cardiovasculares, las drogodependencias, etcétera. La paradoja estriba en que detrás de ellas aparece un sujeto irresponsable cuando en muchos casos el origen causal tiene que ver con la escala de valores predominante. Esta contradicción es evidente en el análisis del patrón de conducta tipo A. En dicho patrón comportamental la ansiedad puede ser un rasgo de personalidad, pero en muchos casos viene propiciado por una cultura laboral competitiva.

Además, estos cambios se traducen en la crisis del sistema sanitario en el Estado de Bienestar y en la necesidad de priorizar en sus servicios las necesidades sociales y la participación ciudadana (San Martín, 1982). Una orientación exclusivamente medicalizada $\mathrm{y}$ curativa de los servicios de salud, resulta insuficiente para abordar el tema de la calidad de vida y el bienestar de las personas.

En este sentido, desde los trabajos de Lalonde (1974) en Canadá, en los que se definían los determinantes de la salud y se subrayaba la importancia de los factores sociales en la mejora y el mantenimiento del bienestar, esta sensibilización social se ha plasmado en diferentes reglamentaciones interna- 
cionales. Especialmente importante sería la filosofía subyacente al plan "Salud para todos desde la OMS (1986)": la trascendencia de políticas centradas en la modificación de los estilos de vida, la introducción de cambios medioambientales y sociales se constituye en una declaración de principios recogida en numerosas directrices comunitarias.

En Europa, como expone Villalbí (1994), el tratado de Maastricht supone una apuesta explícita por la prevención y por la subsidiaridad, o lo que es lo mismo, el reconocimiento de la necesidad de intervenir a un nivel lo más cercano posible a las causas subyacentes de los patrones de enfermedad.

\section{El ESTRÉS}

La inclusión de variables psicosociales como los estilos de vida en las investigaciones e intervenciones sobre la salud no es una aportación reciente. Podemos remontarnos a los padres de la sociología y a los estudios de Durkheim (1951) sobre las transformaciones de la sociedad industrial: los incipientes núcleos urbanos, al disminuir la cantidad y calidad de las relaciones personales, reducen el apoyo social y, por tanto, una de las principales fuentes de bienestar. En 1976 Cassel revisa cómo el ambiente social contribuye a potenciar o debilitar la capacidad inmunológica del organismo.

En la interacción entre indicadores psicosociofisiológicos y la importancia del ambiente como agente causal de la vulnerabilidad, las teorías sobre el estrés son marcos de referencia importantes en el diagnóstico de la salud comunitaria. Como ejemplo del impacto de estos marcos técnicos en la investigación, en 1976 Selye contabilizó 95 mil publicaciones sobre el tema; en 1989 Renaud encontró que este número se había duplicado.

Rodríguez Domingo (1991: 59), en un intento de clasificar las posibles conclusiones de este cúmulo de estudios, los sitúa en tres tipos de categorías:

- El estrés se plantea como una variable dependiente, el énfasis se coloca en la respuesta ocasionada por una situación de riesgo (Glaser, 1987). La dificultad de este modelo estriba en que de manera redundante se define como situación de riesgo toda aquella que produce el síndrome del estrés.

- La definición de estrés como variable independiente soluciona esta carencia, delimitando las características del estímulo producido por la situación de riesgo. Se relacionan los eventos vitales; por ejemplo, la pérdida del empleo con una disminución de la respuesta inmunológica (Cohen, 1986).

- Un tercer grupo de teorías toma en consideración las diferencias individuales y define estrés como una respuesta adaptativa o disfuncional entre la persona y su ambiente. Powel (1987) destacaría entre estas líneas de investigación las que estu- 
dian los patrones de conducta tipo A y B. Lazarus $(1966,1984)$ introduciría la influencia de la percepción cognitiva de la situación y los recursos disponibles para solventarla como elementos mediadores entre el ambiente y el comportamiento individual.

\section{El Patrón de conducta tipo A}

En el análisis psicosocial de los estilos de vida podemos hablar de patrones de conducta que predominan de forma homogénea, independientemente de las diferencias individuales (Mendoza, 1990). De hecho, se halla semejanza entre estas pautas comportamentales en sociedades similares aunque geográficamente distantes (por ejemplo, en Austria y Gran Bretaña). De este modo, entre los retos de un mercado laboral complejo encontramos la primacía de la competitividad y recompensa (logro) del trabajador. Esta situación fuerza al individuo a una carrera contra el reloj en la que prima la productividad (Dossey, 1986). Así, podemos concluir que determinados entornos laborales son estresantes por diferentes causas sociales; por ejemplo, innovaciones impuestas, decisiones unilaterales y arbitrarias, clima laboral enrarecido, criterios injustos de evaluación y desempeño, etcétera.

Estas situaciones objetivas que generan patrones estandarizados de comportamiento han de complementarse con la percepción que la persona (en este ejemplo, el trabajador) tenga de su realidad y determinadas características de personalidad que pueden confluir en una mayor vulnerabilidad en la percepción de situaciones estresantes.

En este sentido, el patrón de conducta tipo A se constituye en una mezcla compleja de estos factores. Definiría a aquellas personas que tienen una forma de vida competitiva con una gran implicación en el trabajo o con una gran sensación de urgencia en las actividades que realizan y que presentan una mayor probabilidad de desarrollar enfermedades coronarias e infarto de miocardio en particular (MacDougall et al., 1985).

Desde esta visión multidimensional, en el patrón de conducta tipo A deberíamos destacar tres características (Friedman y Rosenman, 1974):

- Orientación de logro competitivo.Las personas con este patrón de conducta tienden a ser muy autocríticas y a esforzarse mucho por conseguir sus metas, sin acompañar sus esfuerzos con sentimientos de alegría. Perciben el entorno como opuesto a sus objetivos, con un nivel de reto elevado, interpretan el contexto de forma amenazante para su autoestima y necesitan autoafirmarse y recuperar la sensación de control a través de sus logros personales.

- Urgencia temporal.- Estas personas parecen estar luchando permanentemente contra el tiempo, son muy in- 
tolerantes con los retrasos, con las pausas improductivas e intentan hacer más de una cosa a la vez.

- Sentimientos de ira y hostilidad, que no necesariamente se manifiestan explícitamente pero que definiríamos como el factor más pernicioso. De acuerdo con los estudios al respecto (Rodríguez, 1995), las personas con este patrón de conducta responden más rápidamente y con más fuerza a los estresores, interpretándolos frecuentemente como amenazas a su control personal. Además, el propio patrón comportamental puede incrementar la probabilidad de percepciones estresantes (Byrne y Rosenman, 1986).

\section{INSTRUMENTOS DE EVALUACIÓN}

Con el fin de facilitar la intervención, en primer lugar y centrándonos en la población susceptible de tratamiento, resulta imprescindible realizar un diagnóstico adecuado de las necesidades y factores tanto ambientales como individuales preexistentes:

A nivel individual, destacamos dos aspectos:

- Evaluación de los factores de riesgo o vulnerabilidad que presenta una persona, en relación con los ya anteriormente descritos y estiman su incremento, ya que a mayor número de factores, mayor incidencia de riesgo.

- Intervención preventiva: la evaluación pondría de manifiesto las áreas donde es necesario hacer explícita una intervención preventiva para un sujeto concreto.

A nivel colectivo se pueden citar la promoción de la salud, la evaluación y la formación.

En cuanto a los factores de riesgo, destacaríamos los factores inherentes y los emocionales.

1. Factores inherentes y tradicionales, que actuarán como factores de los que dependerán el resto de los factores de riesgo, haciendo más crítica la intervención preventiva. Un ejemplo de las tendencias sociales se centra en el siguiente perfil, constatado por la evidencia empírica:

- Sexo: los hombres tienen más riesgo que las mujeres.

- Edad: a mayor edad, mayor riesgo (60 años).

- Antecedentes.

- Niveles de colesterol en la sangre $(220 \mathrm{mg} / \mathrm{dl})$ y niveles de presión arterial $(135,6 \mathrm{~mm} / \mathrm{kg})$.

- Peso.

- Hábitos relacionados con el tabaco.

- La recogida de esta información se hará a través de determinaciones clínicas, analíticas, determinación de la presión y entrevistas.

2. Factores emocionales.- Este tipo de evaluación nos lleva a recabar información acerca del patrón de este tipo A, el conglomerado emocional de ira y hostilidad, y el apoyo social. 
- Patrón de conducta tipo A, se dispone de un considerable número de instrumentos de medida que muestran cierta capacidad de predicción del riesgo coronario:

- Entrevista estructurada (Rosenman et al., 1964).

- Inventario de actividad de Yenkin (Yenkin, Zyacumskin y Ronsenman (1979).

- Escala de tipo A de Framinghan (Haynes et al., 1980).

- Escala valorativa de Bartner (Bartner y Rosenman, 1967).

Pero como de entre ellos el instrumento más utilizado en el campo preventivo es el Jenkins Activity Survey (JAS), recomendamos el uso de la relación entre la puntuación central de la adaptación española (JAS; Fernández-Abascal, 1992) y el porcentaje de eventos coronarios, produciéndose a partir del centil 50, un marcado incremento en la vulnerabilidad a sufrir accidentes de esta índole.

3. Factor de ira y hostilidad. También son muchos los instrumentos de medida disponibles. No obstante, a pesar del considerable esfuerzo, dada su importancia preventiva para la creación de medidas fiables de ira y hostilidad, encontramos un problema de validez en la mayoría de ellas, considerando su reciente implantación. Por ello, de entre todos ellos hemos seleccionado:
- En primer lugar, el más validado: la entrevista estructurada que evalúa las dimensiones de potencial de hostilidad, de ira dirigida hacia fuera y hacia dentro.

- En segundo lugar, el más utilizado por su aplicación colectiva: el Inventario de Hostilidad de Buss-Durkee, que proporciona una medida global de hostilidad y siete subescalas: agresión física, agresión indirecta, irritabilidad, resentimiento, negativismo, sospecha y hostilidad verbal.

- Apoyo social. De igual forma, se disponen diversos instrumentos y cualquiera de ellos puede ser adecuado, aunque el Inventario Comportamental de Soporte Social proporciona una información más útil para las aplicaciones clínicas. Además se han utilizado entrevistas como la Interview Schedule for Social Interaction de Henderson, Duncan-Jones, Byne y Scott (1980) y la Arizona Social Support Interview Schedule de Barrera (1980).

En conclusión, una versión abreviada de diagnóstico debería contemplar como mínimo los siguientes instrumentos de medida:

- Entrevista estructurada, observación de campo, técnicas de investigación social para la aproximación a una realidad objetiva y subjetiva.

- Escala de actividad de Yenkims en su adaptación española de 
Fernández Abascal (JAS) y el Inventario de Hostilidad de Buss-Durkee.

- Esta evaluación puede ampliarse con el análisis de las creencias básicas de la persona y de su entorno laboral, como, por ejemplo, el valor de la productividad, la competitividad, el miedo al fracaso, ya que pueden ser importantes al igual que todos los aspectos fisiológicos en la dimensión de la respuesta emocional.

\section{ESTRATEGIAS DE INTERVENCIÓN}

La intervención preventiva de la enfermedad coronaria y del correspondiente patrón de conducta tipo A, debe incluir necesariamente actuaciones, tanto sobre los factores de riesgo tradicional como sobre los emocionales. Y dada la especificidad de los tradicionales, es decir que no todos los factores están presentes en un sujeto en concreto, la intervención dependerá de los aspectos encontrados en la evaluación de cada sujeto. De manera complementaria, la intervención sobre los elementos emocionales parece tener unos efectos más generales en el bienestar del individuo. En cualquier caso, sobre la base de los datos epidemiológicos, etiológicos y de eficacia disponibles, es necesario incidir en la necesidad de una intervención globalizada que afecte los diversos riesgos que presente un sujeto o un colectivo en particular, para el que se desarrollará el programa.
Por otra parte, es necesario señalar (Fernández-Abascal, 1992) que los esfuerzos dirigidos a modificar los factores de riesgo mediante enfoques exclusivamente educacionales, no han mostrado ser suficientemente efectivos y, por el contrario, la utilización de programas comportamentales, con técnicas que garanticen la modificación de conducta es la alternativa eficaz para ayudar a la gente a cambiar los estilos de vida y los comportamientos (por ejemplo: reducir las grasas saturadas en la dieta, dejar el hábito de fumar o la adherencia a regímenes antihipertensivos, etc.); por tanto, reducir la propensión a la enfermedad coronaria.

Por último, centrándonos en la intervención del patrón de conducta tipo A y en el constructo de ira y hostilidad que es nuestro objeto de estudio, el principal fin ha de ser reducir el uso de estrategias de afrontamiento activo y su sustitución por técnicas alternativas que reduzcan la tensión y eliminen los elementos patógenos que conlleva el enfrentamiento activo.

Históricamente, se han seguido los siguientes programas:

- Programa de administración de estrés cardiaco (Suinn, 1974). Duración limitada, nueve horas de intervención. Su misión es enseñar a discriminar las consecuencias psicológicas y fisiológicas de la ansiedadtensión.

- Proyecto de prevención coronaria periódica de Friedman \& Rosenman. 
$\mathrm{Su}$ objetivo es modificar los componentes fisiológicos, comportamentales y cognitivos asociados al tipo A, utilizando un promedio superior de cien horas, repartidas en un período de tres años. El programa parece obtener sus efectos máximos a partir del primer año de entrenamiento.

- Programas de duración media, con un promedio de treinta horas de entrenamiento, como el programa de conducta del proyecto Montreal de Roskies, Sereganian, Oseasohn, Hanley y Collu (1986), que consta de relajación, habilidades de comunicación, reestructuración cognitiva e inaculación de estrés; o la terapia multimodal de comportamiento de Jenni y Wollersheim (1979), que se compone de reestructuración cognitiva, desensibilización del estrés y manejo conductual.

Pero independientemente del tipo de programa utilizado, la gama de técnicas que se han propuesto para reducir el riesgo emocional del patrón tipo A, que presentan un efecto terapéutico mayor, son:

- La educación del riesgo tipo A.- Sesiones educativas en las que se asocia al comportamiento tipo A la enfermedad coronaria, responsable de un $39 \%$ de los efectos positivos.

- La reestructuración cognitiva.Identificación de los errores de pensamiento, así como creencias irracionales y su posterior modificación mediante reestructuración. Aporta el 37\% de los efectos positivos.

- Imaginería.- Que consiste en imaginar situaciones de alta activación, las cuales son utilizadas para practicar habilidades específicas de afrontamiento, desarrolladas mediante relajación o reestructuración. Explican el 21\% de los resultados positivos.

- Relajación.- Procedimientos como la relajación progresiva o el yoga, con beneficios de un $18 \%$.

- Afrontamiento tipo B.- Basado fundamentalmente en la técnica del rolplaying, que tiene como finalidad aprender estrategias alternativas a las manifestadas por el patrón tipo A. Explican el $15 \%$ de los resultados.

\section{MODELO DE COMPETENCIA}

Desde los orígenes de la psicología comunitaria, se ha dado una gran importancia a que los niveles de intervención psicosocial trasciendan el marco clínico y tengan propiedades preventivas (Heller et al., 1984). Se configura así el modelo de competencia (Albee, 1980; Costa y López, 1983, 1986), cuya aproximación a la salud persigue la consecución de dos objetivos: promover el control y la responsabilidad individual, y desarrollar comunidades y organizaciones saludables.

Como hemos visto, la incidencia de los problemas de salud es una razón variable que depende, por una parte, de 
las causas orgánicas, de las circunstancias estresantes de la vida y de las necesidades en general. Holmes y Rahe (1967) plantean que la frecuencia de cambios en los patrones habituales de vida está asociada tanto con el bienestar como con la enfermedad. La prevención parece ser la consensuada piedra angular (Coddington, 1972; Doherenwend \& Dohrenwend, 1972; Skinner \& Lei, 1980; Stern, McCants \& Pethine, 1982; Toro, Font \& Canalda, 1983).

Por todo lo expuesto, podríamos decir que un estilo de vida con características similares a las anteriormente comentadas constituiría un problema de índole psicosocial y médico (alta incidencia en los criterios de morbilidad y epidemiología) y supone un motivo importante para que las instituciones, servicios y otros organismos lo consideren prioritario a la hora de planificar estrategias dirigidas a estos grupos de población afectada, necesitada y condicionada.

La planificación sobre modificación de estilos de vida estaría dirigida:

- Al sector de la población cuyo desempeño profesional y laboral presenta una mayor probabilidad de desarrollar dichos patrones (por ejemplo, el comportamiento propio de ejecutivos, directivos, empresarios con altas responsabilidades).

- A los medios laborales donde se fomente la competitividad individual, poniendo en marcha el mayor nú- mero de recursos, tanto humanos y materiales como financieros, para la pertinente prevención e intervención.

La base de dicha intervención desde un modelo de competencia es sencilla: las personas pueden aprender a controlar el comportamiento tipo A, aunque este tenga bases fisiológicas (Chesney et al., 1985; Powell et al., 1984). De este modo, la competencia personal o afrontamiento (Cohen \& Lazarus, 1979; Mira, 1990) se define como el conjunto de recursos y esfuerzos que hacen los individuos para manejar las demandas del entorno, pudiendo cumplir dos funciones (Cohen, 1987; Pearling \& Schooler, 1978):

- Resolución de problemas (encauzar las demandas internas o ambientales que crean la amenaza, por ejemplo estudiar para un examen).

- Regulación de la emoción (esfuerzos para modificar el malestar que acompaña la amenaza).

Folkman \& Lazarus (1986) señalarían los siguientes recursos preventivos con los que cuenta el individuo para encauzar situaciones potencialmente estresantes:

- Materiales y económicos: Acceso fácil a toda clase de asistencia.

- Vitales: Salud y energía.

- Psicológicos: Creencias generales y expectativas de control sobre la situación y la emoción específica.

- Técnicas de resolución de proble- 
mas: Obtener información sobre el problema, analizar situaciones, prever posibles alternativas, elegir un plan de acción idóneo.

- Habilidades sociales: Implican la capacidad para comunicar e interactuar con los demás de modo adecuado, facilitan la solución de los problemas y el apoyo social.

- Apoyo social: Implica disponer de ayuda, comprensión, consejo e incluso apoyo tangible.

- Culturales (añadida por Rodríguez, 1995): Creencias de la persona y procesos de atribución causal que proceden de un entorno institucional (por ejemplo: lugar del trabajo).

\section{Planificación de un PRograma PREVENTIVO}

Por tanto, cualquier programa preventivo sobre estilos de vida, al considerar estos recursos, debe superar por lo menos algunos errores implícitos y frecuentes en nuestras creencias cotidianas (Gil-Lacruz, 1997):

- Se supone que el nexo entre la información y el comportamiento es automático y eficaz. Por ejemplo, el aumento del nivel educativo no supone una disminución de los comportamientos de riesgo.

- Se supone que el individuo es un ser ahistórico que ejecuta su conducta sin interactuar con su medio físico y social, y, sin embargo, dicha rela- ción en el patrón tipo A es una realidad disfuncional incuestionable.

Por ello, el equipo interdisciplinar profesional de la salud ante un programa preventivo sobre este tema, debe incidir en los siguientes niveles de análisis e intervención:

- Microsocial: Mediante la aplicación de las teorías del aprendizaje y del cambio actitudinal a la conducta de los individuos. En este artículo hemos expuesto algunas de las estrategias de modificación de conducta más extendidas: reestructuración cognitiva, imaginación y afrontamiento.

- Macrosocial: A través de la introducción de cambios estructurales más allá del comportamiento individual y utilizando las estrategias políticas de acercamiento a las esferas de poder sociopolítico.

La planificación de un programa de salud que busque mejorar las respuestas de afrontamiento debe considerar diferentes criterios de clasificación de dichas alternativas, con el fin de facilitar su operativización en variables diana (Rodríguez, 1995).

El punto clave sobre el que se debería intervenir se podría definir como la potenciación de los recursos de competencia o amortiguadores del estrés (Peiró \& Salvador, 1993); entre estos: 
Tabla 1

Taxonomía de las respuestas de afrontamiento

\begin{tabular}{ll}
\hline & Orientadas en la emoción \\
Según el foco & Orientadas en el problema \\
& Orientadas a la situación \\
& Orientadas a la representación \\
& Orientadas a la evaluación \\
& Aproximativas \\
Según el método & Pasivas \\
& Evitativas \\
Según el tipo de proceso & Comportamentales \\
& Cognitivas \\
Según el momento & Anticipatorias \\
& Restaurativas \\
Según su amplitud & Generales / globales \\
& Específicas \\
\hline
\end{tabular}

1. Características personales: Desarrollo de un puesto de trabajo individualizado, destacando dos grupos de variables que se deben considerar dentro del propio capital humano:

- Tolerancia al estrés: Diferentes formas de experiencias estresantes, como la anticipación del cambio, el período de implantación de innovaciones y la adaptación a largo plazo, lo que supone la disponibilidad de recursos de afrontamiento.

- Variabilidad cognitiva: En la que disponemos de capacidades de procesamiento de la información, resolución de problemas y capacidad de ejecución de la tarea.
2. Participación de los usuarios en el diseño del trabajo, facilitando sentimientos de mayor control e implicación, un mejor ajuste del diseño del sistema a las demandas del usuario y acceso a mayor información, que representa cierta forma de entrenamiento (Mumford, 1979).

3. Entrenamiento: Los efectos minimizadores del estrés derivados del entrenamiento pueden ser considerados a corto plazo (una mayor autoconfianza y una actitud positiva que disminuye los miedos y la ansiedad) y largo plazo (no garantiza la transferencia de habilidades al puesto de trabajo, es necesario buscar situaciones directas). Un programa de esta índole debería planificar las estrategias de: 
- Información acerca de:

- Qué es el estrés, marco conceptual.

- Diferentes estilos de vida y de formas de afrontamiento.

- Relación entre pensamiento, emoción y acción.

- Información sobre técnicas y métodos.

- Fomento de actitudes: anticipación de consecuencias positivas a corto plazo.

- Aumento del rendimiento con menor coste y de forma más relajada.

- Mejores relaciones interpersonales.

- Descenso de enfermedades psicosomáticas.

- Aumento de la calidad de vida.

De cara a la intervención, este entrenamiento se podría materializar en diferentes tipos de acciones:

- Cursos de formación dirigidos por profesionales de la salud mental.

- Modificación de respuestas de los trabajadores con técnicas de afrontamiento.

- Cambio de aquellos elementos del entorno laboral, que provocan consecuencias estresantes.

4. La evidencia empírica se ha centrado en la importancia de las interacciones frente a las situaciones de vulnerabilidad o aislamiento. Sus funciones específicas en el individuo como amortiguador del estrés son (Jung, 1990): permiten compartir problemas comunes, proporcionando intimidad, previniendo del aislamiento, definiendo las competencias mutuas, proporcionando elementos de referencia y ofreciendo asistencia en las crisis. En este sentido, sería especialmente importante sensibilizar a los responsables de contextos empresariales y comerciales, entre otros, acerca de la importancia de adoptar medidas para la prevención y, en último término, la intervención, y persuadir a organismos e instituciones para que aporten fondos y medidas operativas que supongan una toma de conciencia por parte de la población general sobre un problema que, además de individual, tiene un importante componente social y cultural. 


\section{REFERENCIAS}

Albee, G. W. (1980). A competency model to replace the defect model. En: M. Costa \& E. López (1986). Salud comunitaria. Barcelona: Martínez Roca.

Barrera, G. (1980). Estrés y prevención coronaria. En: J. M. Buceta \& A. M. Bueno. Psicología y salud. Control del estrés y trastornos asociados. Madrid: Dykinson (1995).

Bartner (1967). Estrés y prevención coronaria. En: J. M. Buceta \& A. M. Bueno. Psicología y salud. Control del estrés y trastornos asociados. Madrid: Dykinson (1995).

Buss-Durkee. (1957). Estrés y prevención coronaria. En: J. M. Buceta \& A.M. Bueno. Psicología y salud. Control del estrés y trastornos asociados. Madrid: Dykinson (1995).

Byrne, D. G. \& Rosenman, R. H. (1986). The type A behavior pattern as a precursor to stressful life events: A confluence of coronary risks. British Journal of Medical Psychology, 59, 75-82.

Cassel, J. C. (1976). The contribution of social environment to host resistance. American Journal of Epidemiology, 104, 107-123.

Chesney, M. A.; Frautschi, N. M. \& Rosenman, R. H. (1985). Modification of type A behavior. En: L. Davidoff (1989). Introducción a la Psicología. Madrid: McGraw-Hill.

Coddington, R. D. (1972). The significance of life events as etiologic factors in deseases of children. A survey of professional workers. Journal of Psychosomatic Research, 16, 7-18.
Cohen, S. (1986). Behavior, health and environmental stress. Nueva York: Prenum Press.

Cohen F. (1987). Measurement of coping. En: J. M. Mira (1990). Psicología y salud. Valencia. Promolibro.

Cohen, F. \& Lazarus, R. S. (1979). Coping with the stresses of illnes. En: J. M. Mira (1990). Psicología y salud. Valencia: Promolibro.

Costa, M. \& López, E. (1983). Aportaciones de la ciencia del comportamiento en un Servicio Nacional de Salud. Papeles del Colegio, 9, 11-21.

Costa, M. \& López, E. (1986). Salud. Barcelona: Martínez Roca.

Doherenwend, E. S. \& Doherenwend, B. P. (1972). Stressful life events: Their nature and effects. Nueva York: Wiley.

Dossey, L. (1986). Tiempo, espacio y medicina. Barcelona. Kairos.

Durkheim, E. (1951, trad.). Suicide: A study in sociology. Nueva York: The Free Press.

Fernández-Abascal, E. (1992). Estrés y prevención coronaria. En: J. M. Buceta \& A. M. Bueno. Psicología y salud. Control del estrés y trastornos asociados. Madrid: Dykinson (1995).

Folkman, S. \& Lazarus, R. S. (1986). Estrés y procesos cognitivos. Barcelona: Martínez Roca.

Framighan (1995). Estrés y prevención coronaria. En: J. M. Buceta \& A. M. Bueno. Psicología y salud. Control del estrés y trastornos asociados. Madrid: Dykinson (1995).

Friedman, M. \& Rosenman, R. H. (1974). Type A behavior and your heart. Nueva York: Knopf. 
Gil-Lacruz, M. (1997). Salud y fuentes de apoyo: Una relación contextualizada. Tesis doctoral. Universidad de Valencia, Facultad de Psicología.

Glaser, R. et al. (1987). Stress related inmune supresion: Health implication. En: E. Rodríguez Domingo (1991). Las teorías del estrés como discurso ideológico. Anthropos (Sociedad urbana y salud), 118-9, 58-62.

González Moro, V. (1990). Los estilos de vida y la cultura cotidiana: Un modelo de investigación. San Sebastián: Baroja.

Haynes, S. N. (1978). Principles of behavioral assessment. Nueva York: Gardner Press.

Henderson, Duncan-Janes; Byne \& Scott (1980). Estrés y prevención coronaria. En: J. M. Buceta \& A. M. Bueno. Psicología y salud. Control del estrés y trastornos asociados. Madrid: Dykinson (1995).

Heller, K.; Price, R. H.; Reinharz, S.; Riger, S. \& Wandersman, A. (1984). Psychology and community change. Challenges of the future. Illinois: Dorsey.

Holmes, J. H. \& Rahe, R. H. (1967). The readjustment rating scale. Journal of Psychosomatic Research, 11, 213-218.

Jenni \& Wollerrsheim (1979). Estrés y prevención coronaria. En: Psicología y salud. Control del estrés y trastornos asociados. Madrid: Dykinson (1995).

Jenkins (1995) . Estrés y prevención coronaria. En: J. M. Buceta \& A. M. Bueno. Control del estrés y trastornos asociados. Madrid: Dykinson (1995).

Lalonde, M. A. (1974). A new perspective on the health of canadians. Otawa: De- partment of National Health and Welfare.

Lazarus, R. S. (1966). Psychological stress and the coping process. Nueva York: McGraw-Hill.

Lazarus, R. S. \& Folkman, S. (1984). Stress, appraisal and coping. Nueva York: Springer.

Mac Dougall, J. M. et al. (1985). Components of type A, hostility, and anger. En: Further relationships to angiographic findings. Health Psychology, 4, 137-152.

Maestro, A. y Huertas, R. (1992). La salud $y$ el estado: Los servicios sanitarios públicos, entre el bienestar y la crisis. Madrid: Fundación de Investigaciones Marxistas.

Mendoza, R. (1990). Concepto de estilos de vida saludables y factores determinantes. En: S. Barriga (1992). La salud ¿para qué? Revista de Psicología de la Salud, 4, vol. 1, 4-20.

Mira, J. M. (1990). Psicología y salud. Valencia. Promolibro.

Montiel, L. (1993). La salud en el Estado de Bienestar. Análisis histórico. Madrid: Universidad Complutense.

Mumford, E. (1979). Conclusiones. En: J. M. Peiró y F. Prieto (1996). Tratado de psicología del trabajo, vol. 1. La actividad laboral en su contexto. Madrid: Síntesis.

Peiró, J. M. \& Salvador, A. (1993). Control del estrés laboral. Madrid: Eudema.

Peraling y Shooler (1978). The structure of coping. Journal of Health and Social Beahvior, 19, 2-21.

Powell, L. H. (1987). Issues in the measurement of the type A behavior pattern. 
En: E. Rodríguez Domingo (1991). Las teorías del estrés como discurso ideológico. Anthropos (Sociedad urbana y salud), 118-9, 58-62.

Powell, L. H.; Friedman, M.; Thoresen, C. E.; Gill, J. J. \& Ulmer, D. K. (1984). Can the type A beahavior pattern be altered after myocardial infarction? En: L. Davidoff (1989). Introducción a la Psicología. Madrid: McGraw-Hill.

Rodríguez Domingo, E. (1991). Las teorías del estrés como discurso ideológico. Anthropos (Sociedad urbana y salud), 118-9, 58-62.

Rodríguez Marín, J. (1995). Psicología social de la salud. Madrid: Síntesis

Roskies, Sereganian, Oseassohn, Hanley \& Collu (1986). Estrés y prevención coronaria. En: J. M. Buceta \& A. M. Bueno. Psicología y salud. Control del estrés y trastornos asociados. Madrid: Dykinson (1995).

San Martín, H. (1982). La crisis mundial de la salud: Problemas actuales de epidemiología social. Madrid: Karpos.

Skinner, H. A. \& Lei, H. (1980). The multidimensional assessment of stressful life events. The Journal of Nervous and Mental Disease, 168, 535-541.

Stern, G. S.; McCants, T. R. \& Pettine, P. W. (1982). Stress and illness: Controllable and incontrollable life events relative contributions. Personality and Social Psychology Bulletin, 8, 140-145.

Suinn (1974). Estrés y prevención coronaria. En: J. M. Buceta \& A. M. Bueno. Psicología y salud. Control del estrés y trastornos associados. Madrid: Dykinson (1995).
Toro, J.; Font, M. L. \& Canalda, G. (1983). Hechos estresores y trastornos psiquiátricos en la infancia y adolescencia, un estudio piloto. Revista del Departamento de Psiquiatria de la Facultad de Medicina de Barcelona, 10, 429-433.

Varela, J. \& Álvarez-Uría, F. (1989). Sujetos frágiles. Ensayos de sociología de la desviación. Madrid: Fondo de Cultura Económica.

Villalbí, J. R. (1994). Una política de salud pública para la Unión Europea. Gaceta Sanitaria, 8, 153-4. 